\title{
Raman Spectroscopic Analysis of Normal, Dysplastic and Cancerous Oral Mucosa: A Tissue Engineering Approach
}

\author{
S. A. Mian ${ }^{1,2}$, H. E. Colley ${ }^{2}$, M. S. Ullah' ${ }^{1}$, C. Yorucu ${ }^{1}$, and I. U. Rehman ${ }^{1}$ \\ ${ }^{1}$ The Kroto Research Institute, Department of Materials Science and Engineering, University of Sheffield, Sheffield, UK. \\ ${ }^{2}$ School of Clinical Dentistry, University of Sheffield, Sheffield, UK.
}

\begin{abstract}
Head and neck cancer (HNC) is the sixth most common malignancy worldwide. Squamous cell carcinoma, the primary cause of HNC, evolves from normal epithelium through dysplasia before invading the connective tissue to form a carcinoma. Only $5 \%$ of suspicious lesions progress to cancer and diagnosis currently relies on histopathological evaluation, which is invasive and time consuming. A non-invasive, real-time point-of-care method could overcome these problems and facilitate regular screening. Infrared (IR) and Raman spectroscopy (RS) can non-invasively provide information regarding biochemical differences between normal and abnormal tissues. In this study, RS was employed to distinguish between different tissues-engineered models. 3D tissue engineered models of normal, dysplastic and head and neck squamous cell carcinoma (HNSCC) using normal oral keratinocytes, dysplastic (D19, D20 and DOK) and HNSCC cell lines (Cal27, SCC4 and FaDu) were constructed and their biochemical content predicted by interpretation of their spectral characteristics. Spectral features of normal tissue samples were mainly attributed to lipids, whereas, malignant tissue samples were observed to be protein dominant. Visible differences were found between the spectra of normal, dysplastic and cancerous models, specifically in the bands of amide I and III. The spectra of HNSCC models showed a broad and strong peak of amide I instead of the sharp and weak lipid peak in normal models at band centred at $1667 \mathrm{~cm}^{-1}$. A shift at $2937 \mathrm{~cm}^{-1}$ was only observed in DOK, differentiating them from the other tissue types. Principal Component Analysis (PCA) and Cluster Analysis (CA) distinguished noticeable differences between tissues.
\end{abstract}

\section{Keywords Analytical; Biological Raman; Oral Mucosa; Spectroscopy; Tissue Engineering}

\section{INTRODUCTION}

Head and neck cancer (HNC) is the sixth most common malignancy worldwide. Histopathological analysis of surgical biopsies is the gold standard in diagnosing oral lesions. This procedure is highly respected but is associated with certain limitations such as delays in diagnosis due to several appointments, anxiety to patients because of surgical intervention, and only a small area of tissue can be analysed whereas tumour margin detection remains a challenge for surgeons. Early diagnosis would improve the quality of life for the patients therefore a technology capable of providing real time, non-invasive, and accurate diagnosis would revolutionise the field of oncology. Raman spectroscopy (RS) provides an optical spectrum obtained from the incident light and gives information regarding chemical composition, molecular conformations and biochemical changes in biological tissues non-invasively and real time [1].

The aim of this study is to assess the potential of Raman spectroscopy (RS) as a tool to identify the chemical pathway associated with the progression of oral cancer.

\section{MATERIALS AND METHODS}

Tissue engineered (T.E) models of normal mucosa (NOM) and $\mathrm{HNC}$ were generated by seeding $1 \times 10^{\wedge} 6$ normal oral keratinocytes (NOK) or $2.5 \times 10^{\wedge} 5$ HNC cells (Cal27, SCC4, FaDu, D19, D20 and DOK), respectively with $5 \times 10^{\wedge} 5$ normal oral fibroblasts (NOF) onto de-epidermised dermis (DED) (Fig. 1) [2].

After 14 days culture models were fixed, wax embedded and sectioned for histological or RS analysis.

The Raman spectra were recorded using a DXR Raman microscopic system (Thermo Scientific, Madison, WI, USA). The system was equipped with an Olympus BX51 optical microscope and a $532 \mathrm{~nm}$ diode-pumped solid state laser excitation source. Principal component analysis (PCA) was performed over the amide I $\left(1550 \mathrm{~cm}^{-1}-1750 \mathrm{~cm}^{-1}\right)$ and amide III $\left(1200 \mathrm{~cm}^{-1}-1400 \mathrm{~cm}^{-1}\right)$ regions of normal, dysplastic and cancerous data set. Pre-processing comprised of baseline correction and unit vector normalisation.

\section{RESULTS AND DISCUSSION}

Results showed increased intensities of tryptophan, phenylalanine, amide I and amide III are observed as the tissue changes from normal to dysplasia and cancer [3].

$\mathrm{CH}$ region has shown a clear shift in abnormal tissues which might suggest the alterations in lipids as a result of lipid peroxidation associated with progression of disease.

Broad peaks are observed at amide I and amide III regions in cancer model spectra due to protein dominancy as compared to sharp and weak lipid dominant peaks in normal model spectra (Fig. 3) [4].

PCA results have distinguished different tissue types on the basis of biochemical differences amongst them. Amide I and amide III regions have shown a minimum explained variance of $95 \%$.

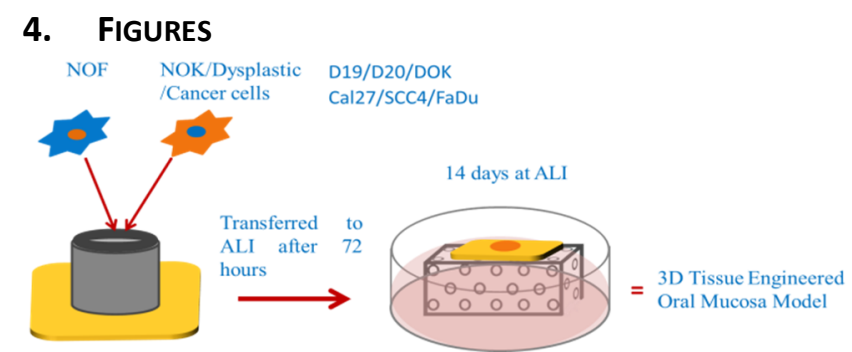

Figure 1. Diagram showing cell seeding onto the DED and inside the chamfered surgical stainless steel ring followed by lifting the model to an air liquid interface (ALI). 


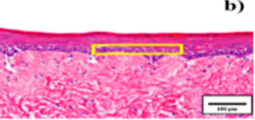

d)
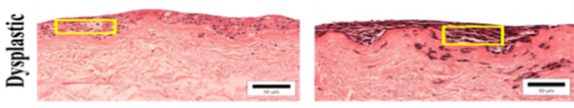

\section{西}

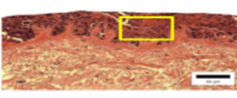

g)

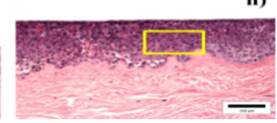

\section{.}

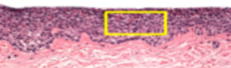

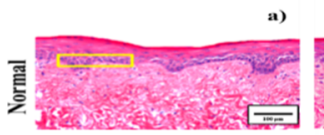

Figure 2. H\&E stained images of tissue engineered oral mucosa; (a-c). Normal oral mucosa; (d-f). Dysplastic oral mucosa, DOK, D19 and D20 respectively; (g-i). Cancerous oral mucosa Cal27, SCC4, and FaDu respectively, Yellow boxes indicate collection points for spectral data on unstained tissues.

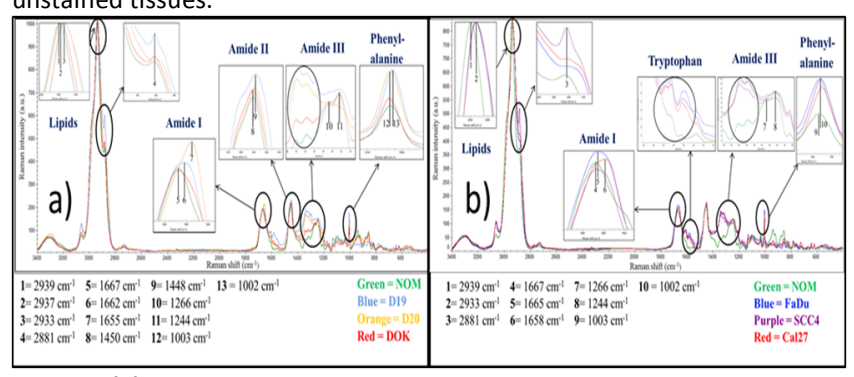

Figure 3(a). Average spectra of tissue engineered normal oral mucosa (NOM) (green) and dysplastic oral mucosa models D20 (yellow), D19 (blue) and DOK (red); (b). Average spectra of tissue engineered normal oral mucosa (NOM) (green) and cancerous oral mucosa models SCC4 (purple), FaDu (blue) and Cal27 (red).
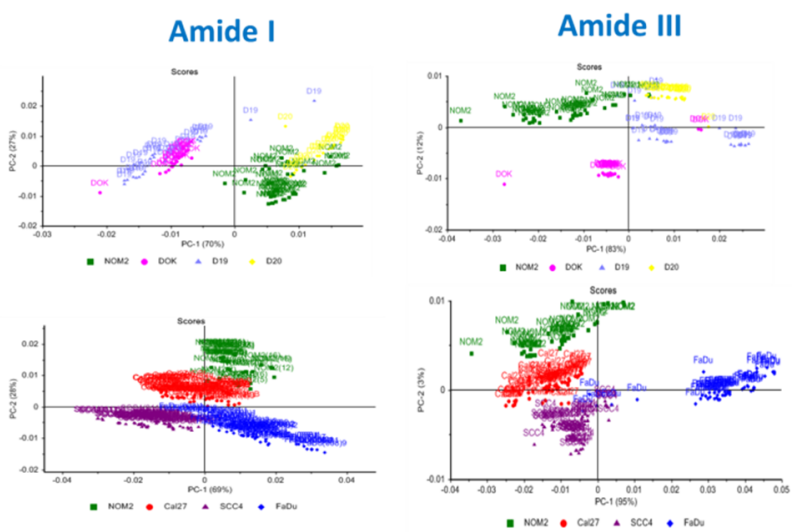

Figure 4. PCA analysis between normal dysplastic and cancer models in amide I and III region.

\section{CONCLUSIONS}

i. RS has the potential to differentiate between normal, dysplastic and cancer tissue models on the basis of their sub-cellular differences.

ii. Compositional changes between the normal and cancerous models showed variations in both peak intensities and positions.

iii. PCA results have shown clearer distinction between T.E normal, dysplastic and cancer models.

\section{REFERENCES}

1. S. Rehman, Z. Movasaghi, A. T. Tucker, S. P. Joel, J. A. Darr, A. V. Ruban and I. U. Rehman, Raman Spectroscopic Analysis of Breast Cancer Tissues: Identifying Differences Between Normal, Invasive Ductal Carcinoma and Ductal Carcinoma in situ of the Breast tissue, Journal of Raman Spectroscopy 38 (2007), no. 10, 1345-1351.

2. H. E. Colley, V. Hearnden, A. V. Jones, P. H. Weinreb, S. M. Violette, S. MacNeil, M. H. Thornhill and C. Murdoch, Development of Tissue-engineered Models of oral dysplasia and early invasive oral squamous cell carcinoma, $\mathrm{Br} J$ Cancer 105 (2011), no. 10, 1582-1592.

3. S. Devpura, J. S. Thakur, S. Sethi, V. M. Naik and R. Naik, Diagnosis of head and neck squamous cell carcinoma using raman spectroscopy: Tongue tissues, Journal of Raman Spectroscopy (2011), n/a-n/a.

4. R. Malini, K. Venkatakrishna, J. Kurien, K. M. Pai, L. Rao, V. B. Kartha and C. M. Krishna, Discrimination of Normal, Inflammatory, Premalignant, and Malignant Oral Tissue: $A$ Raman Spectroscopy Study, Biopolymers 81 (2006), no. 3, 179193. 